\title{
Asymmetric baryon capture by primordial black holes and baryon asymmetry of the Universe
}

\author{
A. D. Dolgov $\circledast^{*}$ and N. A. Pozdnyakov $\circledast^{\dagger}$ \\ Novosibirsk State University Pirogova ul., 2, 630090 Novosibirsk, Russia
}

(Received 26 July 2021; accepted 21 September 2021; published 13 October 2021)

\begin{abstract}
We have refined our previously suggested scenario of generation of the cosmological baryon asymmetry through an asymmetric capture of baryons and antibaryons by a primordial black hole [A. D. Dolgov and N. A. Pozdnyakov, arXiv:2009.04361]. It is found that in the limit of weak interactions of hypothetical heavy baryons with the primeval plasma the effect can be strongly enhanced and the observed magnitude of the asymmetry can be obtained for a wide range of the model parameters.
\end{abstract}

DOI: 10.1103/PhysRevD.104.083524

\section{INTRODUCTION}

The generally accepted mechanism of generation of the cosmological baryon asymmetry was suggested by Sakharov [1] in 1967. Sakharov formulated the following three necessary conditions for the baryogenesis:

(1) Violation of $C$ and $C P$ symmetries in particle physics.

(2) Nonconservation of baryonic number $B$.

(3) Deviation from thermal equilibrium in the early universe.

Out of these three Sakharov's principles the nonconservation of baryons remains yet unconfirmed by experiment, so scenarios of baryogenesis which can operate without assumption of baryon nonconservation are certainly of interest.

The magnitude of the asymmetry expressed in terms of the present-day number densities of baryons, antibaryons, and photons of the cosmic microwave background radiation is equal to (see e.g., review [2])

$$
\beta=\frac{n_{B}-n_{\bar{B}}}{n_{\gamma}} \approx 6 \times 10^{-10}
$$

where $n_{B}$ and $n_{\bar{B}}$ are respectively the number densities of baryons and antibaryons (note that today $n_{\bar{B}} \ll n_{B}$ ), $n_{\gamma}=411\left(T_{\gamma} / 2.73^{\circ} \mathrm{K}\right)^{3} \mathrm{~cm}^{-3}$, and $T_{\gamma}=2.73^{\circ} \mathrm{K}$ is the present-day temperature of the cosmic microwave background radiation.

\footnotetext{
dolgov@fe.infn.it

pozdniko@gmail.com
}

Published by the American Physical Society under the terms of the Creative Commons Attribution 4.0 International license. Further distribution of this work must maintain attribution to the author(s) and the published article's title, journal citation, and DOI. Funded by SCOAP ${ }^{3}$.
There are many different models of baryogenesis which with properly chosen parameters lead to the correct value of the asymmetry; for reviews see Refs. [3-9].

In our scenario excessive antibaryons are hidden inside black holes, so one may say that baryonic number is formally conserved. However, low mass black holes quickly evaporate and disappear from our world without any trace. Thus baryon number is not conserved and the global $U(1)$ symmetry associated with baryonic number is broken. The breaking of all global symmetries, including that related to baryon number, by black holes has been known for a long time. In particular, this breaking can lead to proton instability [10]. Though the lifetime with respect to this decay is fantastically long, $\tau_{p} \sim 10^{45}$ years, one has to admit that B-conservation in particle physics does not save the proton life. In the $\mathrm{TeV}$ gravity [11] a proton might decay almost instantly but the spin and electric charge of a proton could suppress the virtual black hole $(\mathrm{BH})$ formation and very strongly increase the proton lifetime [12].

The idea that black hole evaporation might lead to different numbers of particles and antiparticles in the outer world belongs to Hawking [13], while Zeldovich [14] proposed a concrete mechanism of its realization. Possible generation of the cosmological baryon asymmetry in the process of primoridal black hole ( $\mathrm{PBH}$ ) evaporation and an estimate of its magnitude are described in the subsequent publications $[15,16]$. The calculations of the baryon asymmetry of the universe in the frameworks of the Zeldovich scenario [14] have been performed in Refs. [17,18].

These pioneering works were followed with a plethora of scenarios according to which cosmological baryon asymmetry could be generated in the process of black hole evaporation [19-29]. In contrast to them the model presented in this paper is based on the novel idea that baryogenesis could proceed in the process of asymmetric capture of baryons and antibaryons by primordial black holes. 
Here the ideas suggested in our previous paper [30] are further developed. In Ref. [30] we considered slow diffusion of heavy baryons and antibaryons to primordial black holes because of their short mean free path in the primeval plasma. We have shown that a nonzero value of the baryon asymmetry may be generated in this process, though a strong fine-tuning was necessary to get a reasonable result.

Here we consider the scenario with a large mean free path of the accreting baryons and because of that the model becomes much less constrained allowing for generation of the observed cosmological baryon asymmetry.

The main point of the present work is the difference of mobilities of heavy nonrelativistic baryons in the primeval plasma predominantly consisting of relativistic matter. This difference can be induced by the breaking of $C$ and $C P$ invariance, although $C P T$ remains unbroken in the same way as the partial decay width of particles and antiparticles may be different if a sufficient number of the decay channels are open, see e.g., [31]. A detailed discussion of the cross-section difference is presented in our paper [30]. Hence the accretion rate becomes different and antibaryons can accumulate inside PBHs leaving excess of baryons in our external space.

The paper is organized as follows. In the following section we present and solve the equation of motion of nonrelativistic particles accreting to a central gravitating body (BH) in an expanding Friedmann universe. In Sec. III cosmological baryon asymmetry due to antibaryon capture by a PBH is calculated and the values of the parameters are fixed to ensure the proper magnitude of the asymmetry. Throughout this paper these particles are called $X$ and $\bar{X}$ for baryons and antibaryons respectively. In Sec. IV we describe essential features of $C$ and $C P$ symmetries violation necessary for the implementation of the considered scenario (for more detailed discussion see Ref. [30]). In Sec. V we conclude.

\section{ACCRETION TO BH IN AN EXPANDING UNIVERSE}

Equation of motion (geodesic equation) for nonrelativistic particles, $X$, in the curved background created by a black hole in the Friedmann space-time is derived e.g., in Ref. [32] and has the form

$$
\ddot{r}=\frac{\ddot{a}}{a} r-\frac{r_{g}}{2 r^{2}}+\frac{L^{2}}{r^{3}},
$$

where $r_{g}$ is the Schwarzschild radius, $r_{g}=2 M G_{N} \equiv$ $2 M / m_{\mathrm{Pl}}^{2}, M$ is the $\mathrm{PBH}$ mass, $G_{N}$ is the Newtonian gravitational constant, $m_{\mathrm{Pl}}=1.22 \times 10^{19} \mathrm{GeV}=2.17 \times$ $10^{-5} \mathrm{~g}, L$ is the angular momentum of the $X$ particle, $a(t)$ is the cosmological scale factor, and the overdots mean time derivatives. At the radiation dominated cosmological stage $\ddot{a} / a \approx-H^{2}$ if the PBH does not have an essential impact on the Friedmann expansion. The angular momentum of the $X$ particle $L$ is supposed to be zero since these particles and $\mathrm{PBH}$ are naturally at rest in the comoving frame.

We integrate this equation analytically assuming that $H$ slowly, adiabatically changes with time. As a result we find the following expression for the $X$ particle velocity, $\dot{r}$ :

$$
\dot{r}^{2}=\frac{r_{g}}{r}-H^{2} r^{2}+v_{1}^{2},
$$

where $v_{1}=$ const. We fix this constant by the condition that the particle velocity vanishes at the distance $r=r_{\max }$ corresponding to the equilibrium between the Hubble repulsion and the gravitational attraction induced by the $\mathrm{BH}$ so $r_{\max }$ is the maximum radius of particle capture. As follows from Eq. (3), $r_{\max }$ is equal to

$$
r_{\max }^{3}=\frac{r_{g}}{H^{2}} .
$$

So $v_{1}=0$ and Eq. (3) is solved as

$$
\dot{r}=-\sqrt{\frac{r_{g}}{r}-H^{2} r^{2}}
$$

Equation (5) can be further integrated resulting in

$$
r(t)=r_{\max }[\cos (3 H t / 2)]^{2 / 3} .
$$

It is valid till $r(t)$ drops down to $r_{g}$. Since $r_{g} \ll r_{\max }$, it can be reached at $3 H t / 2$ close to $\pi / 2$. It does not contradict our assumption of slow variation of $H$ which is true by an order of magnitude if $H t \lesssim 1$.

In the free fall approximation there is no difference between the laws of motion for $X$ and $\bar{X}$. To take their difference into account we need to include into equation of motion (2) a small friction term induced by the interaction of $X$ and $\bar{X}$ particles with the plasma surrounding a black hole. With this correction the trajectory of $X$ particles $R(t)$ would obey the equation

$$
\ddot{R}=\frac{\ddot{a}}{a} R-\frac{r_{g}}{2 R^{2}}-\gamma \dot{R},
$$

where $\gamma=\sigma_{e l} v n_{\text {rel }}, \sigma_{e l} \sim f^{4} / m_{X}^{2}$ is the cross section of elastic $X$-particle scattering by the relativistic ones in cosmic plasma, $f$ is the coupling constant, $v \approx 1$ is the relative velocity of $X$ and a relativistic scatterer, and $n_{\text {rel }} \approx$ $0.1 g_{*} T^{3}$ is the number density of all relativistic species in plasma, $g_{*} \approx 100$ is the number of relativistic species, and $T$ is the plasma temperature. Due to $C$ and $C P$ violation $\gamma$ should be different for $X$ and $\bar{X}$, i.e., $\Delta \gamma=\gamma_{X}-\gamma_{\bar{X}} \neq 0$.

In what follows we assume that the ratio $\gamma / H$ is small, although it is not obligatory. The Hubble parameter at the expansion stage dominated by relativistic matter can be expressed through the temperature applying the set of the following equations: 


$$
\varrho=\frac{3 H^{2} m_{\mathrm{Pl}}^{2}}{8 \pi}=\frac{3 m_{\mathrm{Pl}}^{2}}{32 \pi t^{2}}=\frac{\pi^{2} g_{*}}{30} T^{4} .
$$

Hence we obtain

$$
H=\left(\frac{8 \pi^{3} g_{*}}{90}\right)^{1 / 2} \frac{T^{2}}{m_{\mathrm{Pl}}}=16.6 g_{100}^{1 / 2} \frac{T^{2}}{m_{\mathrm{Pl}}},
$$

where $g_{100}=g_{*} / 100$. Comparing the expression for $\gamma$ given above to the obtained value of the Hubble parameter we find that the condition $\gamma<H$ leads to

$$
T<\left(\frac{8 \pi^{3} g_{*}}{90}\right)^{1 / 2} \frac{1}{0.1 g_{*}} \frac{m_{X}^{2}}{f^{4} m_{\mathrm{Pl}}} .
$$

For $g_{*}=100$ and $m_{X}=3 \times 10^{13} \mathrm{GeV}$ we find $T<0.04 m_{X}$.

Assuming that $\gamma / H$ is small, we solve Eq. (7) perturbatively expanding $R$ as $R=r+r_{1}$ where $r$ is the solution of the equation of motion (7) in zeroth order in $\gamma$, so $r(t)$ is given by expression (6). In the first order in $r_{1}$ Eq. (7) is reduced to the following linear inhomogeneous equation:

$$
\ddot{r}_{1}=r_{1} H^{2} \tan ^{2}\left(\frac{3 H t}{2}\right)-\gamma \dot{r}
$$

It is convenient to introduce dimensionless time $\eta=H t$ and to rewrite Eq. (11) in the form

$$
r_{1}^{\prime \prime}=r_{1} \tan ^{2}\left(\frac{3 \eta}{2}\right)-\frac{\gamma}{H} r^{\prime}
$$

where prime means derivative over $\eta$. At short dimensionless time $r^{\prime} \sim r_{\max } \eta$ and $\tan ^{2}(3 \eta / 2) \approx 9 \eta^{2} / 4$, so the first term at the right side of Eq. (12) appears to be smaller than the second one. Hence this equation takes the form

$$
r_{1}^{\prime \prime}=-\frac{\gamma}{H} r^{\prime}
$$

and can be solved as $r_{1}^{\prime}=-\gamma r / H+$ const. We assume here and in what follows that $\gamma$ and $H$ are approximately constant because temperature evolves slower than the essential processes of the capture of $X$ particles by $\mathrm{PBH}$. It is also worth noting that we took the effective time for baryogenesis of the order of $H^{-1}$ so the product $\eta=H t$ always remains smaller than one.

Finally we conclude that the solution for $r_{1}$ satisfying the proper initial condition has the form

$r_{1}^{\prime}=-\frac{\gamma}{H}\left[r_{\max }-r(\eta)\right]=-\frac{\gamma}{H} r_{\max }\left[1-\cos ^{2 / 3}\left(\frac{3 \eta}{2}\right)\right]$, where $r(\eta)$ is the solution (6) with $r_{\max }$, given by Eq. (4), which is supposed to be the initial value of $r(t)$. Expanding the right-hand side of this equation we obtain ${ }^{1}$

$$
r_{1}^{\prime}=-r_{\max } \frac{3 \gamma}{4 H} \eta^{2}
$$

The total number of $X$ or $\bar{X}$ particles captured by a BH during the Hubble time is approximately

$$
N \approx(4 \pi / 3)(1+3 \gamma t) r_{\max }^{3} n_{X},
$$

where $n_{X}$ is the number density of $X$ particles. If the annihilation $X \bar{X}$ is weak (we check below when it is indeed the case) and if $X$ particles are efficiently produced by the inflaton decay at the end of inflation, then

$$
n_{X} \approx n_{\bar{X}} \approx 0.1 g_{s} T^{3},
$$

where $g_{s}$ is the number of spin states of $X$ particles.

Since the $X \bar{X}$ annihilation is weak, the number density of $X$ particles remains unsuppressed even at very small temperatures, $T<m_{X}$.

\section{BARYOGENESIS THROUGH CAPTURE OF BARYONS BY PBHS}

We assume that the heavy particles $X$ and antiparticles $\bar{X}$ have nonzero baryon number $B_{X} \sim 1$. We also assume that there exists an interaction between $X, \bar{X}$, and light particles which breaks $C$ and $C P$ symmetries but respects $C P T$. Some other particles, heavier than $X$ and rather short-lived, are also needed. Their existence is necessary to create the difference between elastic cross sections of $\mathrm{X}$ and $\bar{X}$ particles in the primeval plasma despite the $C P T$ restrictions which demand equality of the total cross sections, see Sec. IV.

The accretion of $X$ particles to a PBH effectively started when these particles became nonrelativistic. As we see in what follows, the smaller the plasma temperature is, the larger the baryon asymmetry is, if the density of the $X$ particles in comoving volume does not drop as $\exp \left(-m_{X} / T\right)$. In other words it happens if the $X \bar{X}$ annihilation froze at temperatures of the order of $m_{X}$. However, such early freezing of massive species, if they are stable, would create too high of a contribution into the cosmological density of dark matter. The problem can be solved if $X$ particles are unstable but live sufficiently long to fulfill their task of creating the baryon asymmetry of the universe. These and some other constraints are considered below in this section.

\footnotetext{
${ }^{1}$ We thank the referee who indicated an inaccuracy of the solution in the previous version of the paper. Nevertheless the final result remains essentially the same.
} 
Using Eq. (16) we can estimate the difference between the number of captured $X$ and $\bar{X}$ particles by a single PBH during time interval $t$ :

$$
\Delta N \approx 4 \pi r_{\max }^{3} n_{X} t \Delta \gamma,
$$

where $n_{X}$ is the number density of $X$ particles after they became nonrelativistic. We should keep in mind that this time duration is bounded by the condition $t \lesssim H(T)$, where $T$ is the favorable temperature for excessive $X$ over $\bar{X}$ capture by PBH, see below.

The difference between the friction coefficients in the case of maximally broken $C$ and $C P$ symmetries can be estimated as

$$
\Delta \gamma=\delta \sigma_{e l} n_{\text {rel }} \approx f^{6} n_{\text {rel }} / m_{X}^{2},
$$

since the cross section of elastic scattering of $X$ particles on the relativistic particles is $\sigma_{e l} \approx f^{4} / m_{X}^{2}$ and the difference between $X$ and $\bar{X}$ scattering is of the order of $f^{2}$ because the cross-section difference appears as a result of radiative correction proportional to $f^{2}$, exactly as there appears the difference between partial decay widths in the scenario of baryogenesis through massive particle decays.

This result is true if the following conditions are fulfilled: the mean free path of $X$ particles in the primeval plasma $l_{\text {free }}$ should be larger than the maximum capture radius $r_{\max }$. The former can be estimated as

$$
l_{\text {free }}=\frac{1}{\sigma_{e l} n_{\text {rel }}}=\frac{m_{X}^{2}}{0.1 g_{*} T^{3} f^{4}},
$$

where $\sigma_{e l}$ and $n_{\text {rel }}$ are defined below Eq. (7).

The condition $l_{\text {free }}>r_{\text {max }}$ can be rewritten as

$$
\frac{m_{X}^{2} H}{0.1 g_{*} f^{4} T^{3}}>\left(r_{g} H\right)^{1 / 3} .
$$

Using Eq. (9), the bound (21) can be rewritten as

$$
M<\frac{0.14 m_{X}^{6}}{g_{100}^{2} f^{12} T^{5}} .
$$

Note that the limit does not depend upon the Planck mass.

If $m_{X}=3 \times 10^{13} \mathrm{GeV}$ (close to the typical heating temperature after inflation), $T=m_{X}$, and $f=0.1$, the condition of the free fall is fulfilled for $M \lesssim 7 \mathrm{~g}$. For higher mass of the PBHs the free fall condition is satisfied at smaller $T$, e.g., if $M=10^{6} \mathrm{~g}$, the efficient free fall capture took place at $T=m_{X} / 10$.

The rate of the annihilation is determined by the equation

$$
\Gamma_{\mathrm{ann}} \equiv \dot{n}_{X} / n_{X}=\sigma_{\mathrm{ann}} v n_{X}=0.1 g_{*} g_{s} f_{\mathrm{ann}}^{4} T^{3} / m_{X}^{2},
$$

where $f_{\text {ann }}$ is the coupling constant of the annihilation. Demanding that $\Gamma_{\text {ann }}$ is small in comparison with $H$, see
Eq. (9), we find that the annihilation would be inefficient at the temperatures satisfying the condition:

$$
\frac{T}{m_{X}}<2.5 \times 10^{-6} f_{\text {ann }}^{-4}\left(\frac{m_{X}}{3 \times 10^{13} \mathrm{GeV}}\right) .
$$

If the maximum value of $T / m_{X}$ may reach unity, then the annihilation does not essentially diminish the density of $X$ particles below $n_{X}=0.1 g_{s} T^{3}$. In other words the density of $X$ and $\bar{X}$ particles would be conserved in the comoving volume, i.e., $n_{X}=0.1 g_{s} T^{3}$ below $T=m_{X}$. For $m_{X}=3 \times$ $10^{13} \mathrm{GeV}$ this could be realized if $f_{\text {ann }} \lesssim 4 \times 10^{-2}$. Otherwise the density of $X$ particles would be exponentially suppressed, $n_{X} \sim \exp \left(-m_{X} / T\right)$ at low temperatures. To avoid an overclosing of the universe by $X$ particles we assume that they are unstable, presumably decaying before the big bang nucleosynthesis. As it was mentioned by the referee, $X$ particles should decay considerably earlier since their huge energy density near BBN could strongly dilute the baryon asymmetry due to a large entropy release. So $X$ particles should decay before their energy density becomes equal to the energy density of relativistic plasma. It happens at $T \approx 0.01 m_{X}$, safely below the process of baryogenesis.

Another important restriction on the efficiency of the mechanism discussed here is that the "size" of an $X$ particle, i.e., its Compton wave length $\lambda_{X}$, should be smaller than the gravitational radius of the PBH. Otherwise the probability of the particle capture would be suppressed by a power of the ratio $r_{g} / \lambda_{c}$ :

$$
\lambda_{X}=1 / m_{X}<r_{g}=2 M / m_{\mathrm{Pl}}^{2} .
$$

It leads to a lower bound on the PBH mass:

$$
M>\frac{m_{\mathrm{Pl}}^{2}}{2 m_{X}}=4.4 \mathrm{~g}\left(\frac{3 \times 10^{13} \mathrm{GeV}}{m_{X}}\right) .
$$

The baryon asymmetry gained by the $\mathrm{PBH}$ antibaryon capture can be diluted by the entropy release from the PBH evaporation. As it follows from Ref. [33] this would be avoided if

$$
\epsilon M<10^{-5} \mathrm{~g},
$$

where $\epsilon$ is the fraction of the energy density of PBHs at the moment of their formation:

$$
\frac{\varrho_{\mathrm{PBH}}\left(t_{\text {form }}\right)}{\varrho_{\text {rel }}\left(t_{\text {form }}\right)}=\epsilon
$$

where $\varrho_{\text {rel }} \approx 3 T n_{\text {rel }}$ is the energy density of the relativistic matter, and

$$
t_{\text {form }}=M / m_{\mathrm{Pl}}^{2} \text {. }
$$

Using Eqs. (8) and (9) we find that the temperature of the relativistic matter at the formation moment is 


$$
\begin{aligned}
T_{\text {form }} & \equiv T\left(t_{\text {form }}\right)=0.17 g_{100}^{-1 / 4} m_{\mathrm{Pl}}\left(\frac{m_{\mathrm{Pl}}}{M}\right)^{1 / 2} \\
& =10^{14} \mathrm{GeV} g_{100}^{-1 / 4} M_{4}^{-1 / 2}
\end{aligned}
$$

where $M_{4}=M / 10^{4} \mathrm{~g}$. For successful baryogenesis PBHs should be created while $X$ particles are abundant in the cosmological plasma. If $X \bar{X}$ annihilation continued till $T \ll m_{X}$, then the temperature of the PBH creation should not be much smaller than $m_{X}$. If, as we assume in the present work, the annihilation of $X \bar{X}$ in thermal plasma was never efficient, then $\mathrm{PBHs}$ should be produced prior to the decay of $X$ (and $\bar{X}$ ) particles.

In the course of the cosmological expansion and cooling down, the energy fraction of a PBH rises as $\left(T_{\text {form }} / T\right)$ until its evaporation, which happens at the time moment equal to the BH life-time $\tau_{\mathrm{BH}}[34]$ :

$$
t=\tau_{\mathrm{BH}} \approx 30 M^{3} / m_{\mathrm{Pl}}^{4} \text {. }
$$

The corresponding temperature is

$$
T\left(\tau_{\mathrm{BH}}\right)=0.03 g_{100}^{-1 / 4} m_{\mathrm{Pl}}\left(\frac{m_{\mathrm{Pl}}}{M}\right)^{3 / 2}=3.7 \times 10^{4} \mathrm{GeVM}_{4}^{-3 / 2} .
$$

The temperature of the relativistic plasma at the moment of PBH decay should be smaller than $m_{X}$ to allow nonrelativistic $X$ particles to be captured by PBHs.

Since PBHs are nonrelativistic, while the bulk of the matter is relativistic, the fraction of the PBH energy density at temperature $T$ becomes larger than $\epsilon$ by the factor

$$
\frac{T_{\text {form }}}{T} \approx g_{100}^{-1 / 4} M_{4}^{-1 / 2} \frac{10^{14} \mathrm{GeV}}{T} .
$$

Now using Eq. (18) and Eq. (19) we find for the excessive baryon number created by a single primordial black hole:

$$
\Delta N=4 \pi f^{6} \frac{r_{g} n_{\mathrm{rel}} n_{X} t}{H^{2} m_{X}^{2}}=\frac{0.9 g_{s} f^{6}}{\pi^{2}} \frac{M T^{2} t}{m_{X}^{2}} .
$$

Hence the baryon asymmetry can be estimated as

$$
\begin{aligned}
\beta & =\frac{B_{X} n_{\mathrm{BH}} \Delta N}{n_{\text {rel }}}=\frac{2.7}{\pi^{2}} B_{X} g_{s} f^{6} \frac{\varrho_{\mathrm{BH}}}{\varrho_{\text {rel }}} \frac{T^{3} t}{m_{X}^{2}} \\
& =\frac{2.7}{\pi^{2}} B_{X} g_{s} f^{6} \epsilon \frac{T_{\text {form }} T^{2} t}{m_{X}^{2}}
\end{aligned}
$$

where $\epsilon$ is the fraction of the PBH energy density to that of the relativistic matter at the moment of their formation, see Eq. (28), $T_{\text {form }}$ is the temperature at PBH formation (30), and $t \sim 1 / H$, so we finally obtain:

$$
\beta \approx 0.016 B_{X} g_{s} f^{6} \epsilon \frac{T_{\text {form }}}{T} \frac{m_{\mathrm{Pl}} T}{m_{X}^{2}} .
$$

Taking the maximum allowed values of $\epsilon$ from Eq. (27) $\epsilon=10^{-5} \mathrm{~g} / M, \quad m_{X}=10^{13} \mathrm{GeV}, \quad f=0.1, \quad T=m_{X} / 10$ $M=10^{4} \mathrm{~g}$ and thus $T_{\text {form }}=10^{14} \mathrm{GeV}$, we find that the baryon asymmetry can easily reach the observed value and even overcome it. This choice of the parameters satisfies the derived above restrictions.

\section{DIFFERENCE BETWEEN MOBILITIES OF $X$ AND $\bar{X}$ PARTICLES IN THE BACKGROUND PLASMA}

In this section we revisit the main concepts from the corresponding section in our previous paper [30]. First, we remind the reader that we assume validity of the first Sakharov condition of violation of $C$ and $C P$ symmetries, while the sacred $C P T$ invariance remains unbroken.

As we have shown in Ref. [30], in this case there should naturally appear a difference between the probabilities of the quasielastic scattering of $X$ particles over relativistic species in the cosmic plasma

$\sum_{a, b} \Gamma(X+a \rightarrow X+b) \neq \sum_{\bar{a}, \bar{b}} \Gamma(\bar{X}+\bar{a} \rightarrow \bar{X}+\bar{b})$,

where summations are done over all light particle sets in the initial state $a$ and the final state $b$.

However, it should be taken into account that according to the $C P T$ theorem the total probability $\Gamma\left[\mathbf{p}_{1}, \lambda_{1}, a_{1}, \mathbf{p}_{2}, \lambda_{2}, a_{2}, \ldots\right]$ of a process from an initial state, containing a certain set of particles is equal to the total probability $\Gamma\left[\mathbf{p}_{1},-\lambda_{1}, \bar{a}_{1}, \mathbf{p}_{2},-\lambda_{2}, \bar{a}_{2}, \ldots\right]$ of the process containing antiparticles with opposite spin projection state, e.g., with opposite helicities $\lambda$ see textbook [35], eq. (3.6.15), or review [3]:

$\Gamma\left[\mathbf{p}_{1}, \lambda_{1}, a_{1}, \mathbf{p}_{2}, \lambda_{2}, a_{2}, \ldots\right]=\Gamma\left[\mathbf{p}_{1},-\lambda_{1}, \bar{a}_{1}, \mathbf{p}_{2},-\lambda_{2}, \bar{a}_{2}, \ldots\right]$.

In particular, this condition leads to the mentioned above equality of the total decay widths of particles and antiparticles while it also allows for a difference between the partial decay rates as well as for a difference among the partial modes of scattering processes, but the magnitude of the latter would be suppressed because the difference may appear only in higher order of perturbation theory.

Then to achieve the desired difference of $X$ and $\bar{X}$ particles scattering we need to introduce a new interaction leading to the disappearance of $X$ and $\bar{X}$ particles through the process of the kind

$$
a+X \rightarrow b+Y,
$$




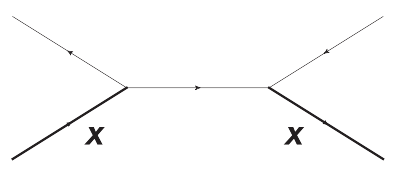

(a)

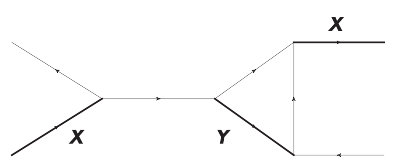

(b)
FIG. 1. Feynman diagrams describing $X$ (or $\bar{X}$ ) scattering off light quark. (a) Lowest order contribution; equal values for particles and antiparticles are shown. (b) Example of the oneloop correction with an exchange of $Y$ particle. The one-loop scattering may be also present in the initial state and its contribution multiplies the result by a factor 2 .

where the heavy particle $Y$ may have zero baryonic number and the light state $b$ should have the same baryonic number as $a+X$, if we want to avoid nonconservation of baryons.

In complete analogy with the higher order corrections to the decay process, which create a difference between the partial decay widths of particles and antiparticles, we consider radiative corrections to elastic scattering of $X$ on relativistic particles, which lead to different values of the cross sections. For more details see our earlier paper [30]. The corresponding Feynman diagrams are presented in Figs. 1(a) and 1(b).

The equalities of the total probabilities means in particular that the total cross section of $X$ scattering on particle " $a$ " $\sigma_{\text {tot }}(X+a \rightarrow$ All $)$ is equal to the same of particles and antiparticles $\sigma_{\text {tot }}(\bar{X}+\bar{a} \rightarrow$ All $)$. If the final state "All" contains one and only one $X$ particle, then the mobilities of $X$ and $\bar{X}$ in the cosmological plasma would be the same and the mechanism of baryogenesis discussed here would not operate. However, if the complete set of the final states includes a state or states where $X$ is missing (and analogously for the reactions with $\bar{X}$ ), then the cross sections of the processes $\sigma_{\text {tot }}(X+a \rightarrow X+$ All $)$ and $\sigma_{\text {tot }}(\bar{X}+\bar{a} \rightarrow$ $\bar{X}+$ All) may be different, leading to the needed mobility differences of $X$ and $\bar{X}$.

The difference between probabilities of charge conjugated processes can be estimated as

$\delta=\frac{\sigma_{X 0}-\sigma_{\bar{X} 0}}{\sigma_{X 0}+\sigma_{\bar{X} 0}} \approx \frac{\left|g_{x 1}\right|^{2} \operatorname{Im}(D) \operatorname{Im}\left(g_{x 1} g_{x 2}^{*} g_{y 1} g_{y 2}^{*}\right)}{\left|g_{x 1}\right|^{4}} \propto f^{2}$,

where $\sigma_{X 0}$ and $\sigma_{\bar{X} 0}$ stand, respectively, for the scattering cross section of $X$ and $\bar{X}$ particles on relativistic particles in cosmic plasma. The complex coefficient $D$ comes from the integration over the loop, and $g_{x i}$ and $g_{y i}$ are partial decay constants of $X$ and $Y$ particles, respectively.

The following supersymmetry inspired model can serve as appropriate frameworks for the scenario. Assume that $X$ is an analog of the lightest supersymmetric particles (LSP) which is stable due to an analog of $R$-parity. Let us assume that there exists a heavier partner $H$ with zero baryonic number which would be unstable and decay through the channel $H \rightarrow X+3 q$, where $q$ are light quarks with proper quantum numbers. Accordingly the reaction $X+q \rightarrow H+2 \bar{q}$ becomes possible. It is exactly what we need to allow for a difference between the cross sections of the reaction $\sigma_{\text {tot }}(X+a \rightarrow X+$ All $)$ and $\sigma_{\text {tot }}(\bar{X}+\bar{a} \rightarrow \bar{X}+$ All $)$, which can lead to different mobilities of $X$ and $\bar{X}$ around a black hole and to dominant capture of antibaryons over baryons creating cosmological baryon asymmetry.

Note that in $R^{2}$ gravity, Srarobinsky inflation [36], the allowed mass of a LSP-kind particle can be close to $10^{13} \mathrm{GeV}$ or even higher [37,38].

\section{CONCLUSION}

In this paper we continue investigation of baryogenesis through the asymmetric capture of baryons and antibaryons by primordial black holes. Unlike in our previous paper [30], where we used diffusion approximation $\gamma / H \gg 1$ in which particles many times scatter off the cosmic plasma before they are captured by $\mathrm{PBH}$, in this study we investigate the opposite limit in which $\gamma / H \ll 1$ or the free fall limit. As it appears, there is a sufficiently wide parameter space to explain the observed value of baryon asymmetry of the universe.

A noticeable increase of the baryon asymmetry generated by the capture of the antibaryonic number by PBH in the considered version of the scenario is achieved due to assumed negligible annihilation of $X$ particles with decreasing temperatures, $T<m_{X}$, because at smaller $T$ the relative fraction of $\mathrm{PBH}$ with respect to the total cosmological energy density goes up quite significantly.

The mechanism of baryogenesis proposed here does not demand two out of three Sakharov's principles. Namely it can proceed in thermal equilibrium and without assumption of nonconservation of baryonic number in particle reactions. It helps to avoid a possible problem which arises because nonconservation of baryons is not (yet) observed in direct experiment.

In a sense black holes break conservation of baryonic number, either hiding baryons in internal space making them unobservable, if black holes are eternal, or transforming an arbitrary amount of baryons into a state with zero baryonic number. For instance a black hole consisting entirely from baryons would completely evaporate creating (almost) an equal number of baryons and antibaryons. In the process of evaporation a small baryon asymmetry might be created but it normally would be negligibly small in comparison with the initial baryonic number captured at the black hole formation.

If baryonic number is conserved in particle interations, the proton must be almost absolutely stable. To be more precise it may decay by Zeldovich mechanism [10] through formation of a virtual black hole from three quarks inside a proton. But the lifetime with respect to such decay is almost 
infinite, $\tau_{p} \sim 10^{45}$ years. Also one could hardly expect neutron-antineutron oscillations induced by virtual $\mathrm{BHs}$ to be observable (for a recent review see e.g., [39]).

Another unusual feature of the model is a possibility to create baryon (or any other type of asymmetry between particles and antiparticles) in thermal equilibrium. Normally the deviation from thermal equilibrium is suppressed by the factor of the order of the ratio of the Hubble expansion rate to the particle reaction rate, $H / \Gamma$. The former is inversely proportional to a huge value of the Planck mass, $H \sim T^{2} / m_{\mathrm{Pl}}$, where $T$ is the cosmological plasma temperature. According to the estimates presented above, for the mechanism considered here the situation is opposite: the larger is the Planck mass (or the slower is the cosmological expansion), the larger is the baryon asymmetry. On the other hand, the gravitational attraction which forces massive $X$ particles to fall on the nearest $\mathrm{BH}$ is inversely proportional to $m_{\mathrm{Pl}}^{2}$, so ultimately the effect disappears in the limit of infinite $m_{\mathrm{Pl}}$ as well.
The magnitude of the baryon asymmetry evidently strongly depends upon the cosmological expansion regime. In particular, it would be very interesting to study baryogenesis in the frameworks of $R^{2}$ inflation [36] where there exists a long period of the universe evolution during which the falloff of the cosmological temperature is drastically different from that accepted in the conventional cosmology [37,38].

In the course of working on the version of baryogenesis presented here we became aware of an interesting modification of the scenario presented in Ref. [30] on the generation of the cosmological baryon asymmetry through the capture of antibaryons by $\mathrm{PBH}$ [40], which also may lead to an efficient baryogenesis.

\section{ACKNOWLEDGMENTS}

This work was supported by RSF Grant No. 20-42-09010. The Feynman diagrams were drawn by JaxoDraw [41].
[1] A. D. Sakharov, JETP Lett. 5, 24 (1967), http://jetpletters.ru/ ps/1643/article_25089.shtml.

[2] P. A. Zyla et al. (Particle Data Group Collaboration), Prog. Theor. Exp. Phys. 2020, 083C01 (2020).

[3] A. D. Dolgov and Ya. B. Zeldovich, Rev. Mod. Phys. 53, 1 (1981).

[4] A. D. Dolgov, Phys. Rep. 222, 309 (1992).

[5] A. G. Cohen, D. B. Kaplan, and A. E. Nelson, Annu. Rev. Nucl. Part. Sci. 43, 27 (1993).

[6] V. A. Rubakov and M. E. Shaposhnikov, Usp. Fiz. Nauk 166, 493 (1996).

[7] A. D. Dolgov, Surv. High Energy Phys. 13, 83 (1998).

[8] A. Riotto and M. Trodden, Annu. Rev. Nucl. Part. Sci. 49, 35 (1999).

[9] J. M. Cline, Proc. Sci., TASI2018 (2019) 001 [arXiv:1807 .08749].

[10] Ya. B. Zel'dovich, Phys. Lett. 59A, 254 (1976); Ya. B. Zel'dovich, Zh. Eksp. Teor. Fiz. 72, 9 (1977) [Sov. Phys. JETP 45, 9 (1977)], http://jetp.ras.ru/cgi-bin/r/index/r/72/1/ p18? $\mathrm{a}=$ list.

[11] N. Arkani-Hamed, S. Dimopoulos, and G. Dvali, Phys. Lett. B 429, 263 (1998).

[12] C. Bambi, A. D. Dolgov, and K. Freese, Nucl. Phys. B763, 91 (2007).

[13] S. W. Hawking, Caltech Preprint (1975) (unpublished).

[14] Ya. B. Zeldovich, Pis'ma Zh. Eksp. Teor. Fiz. 24, 29 (1976), http://jetpletters.ru/ps/1809/article_27646.shtml.

[15] B. J. Carr, Astrophys. J. 206, 8 (1976).

[16] M. S. Turner, Phys. Lett. 89B, 155 (1979).

[17] A. D. Dolgov, Zh. Eksp. Teor. Fiz. 79, 337 (1981), http://jetp .ras.ru/cgi-bin/r/index/r/79/2/p337?a=list.
[18] A. D. Dolgov, Phys. Rev. D 24, 1042 (1981).

[19] E. V. Bugaev, M. G. Elbakidze, and K. V. Konishchev, Yad. Fiz. 66, 504 (2003) [Phys. At. Nucl. 66, 476 (2003)].

[20] Y. Nagatani, arXiv:hep-ph/0104160.

[21] D. Baumann, P. J. Steinhardt, and N. Turok, arXiv:hep-th/ 0703250.

[22] A. Hook, Phys. Rev. D 90, 083535 (2014).

[23] G. Aliferis, G. Kofinas, and V. Zarikas, Phys. Rev. D 91, 045002 (2015).

[24] T. Banks and W. Fischler, arXiv:1505.00472v2.

[25] Y. Hamada and S. Iso, Prog. Theor. Exp. Phys. 2017, 033B02 (2017).

[26] L. Morrison, S. Profumo, and Y. Yu, J. Cosmol. Astropart. Phys. 05 (2019) 005.

[27] L. Di Luzio, M. Redi, A. Strumia, and D. Teresi, J. High Energy Phys. 06 (2019) 110.

[28] A. Boudon, B. Bose, H. Huang, and L. Lombriser, Phys. Rev. D 103, 083504 (2021).

[29] D. Hooper and G. Krnjaic, Phys. Rev. D 103, 043504 (2021).

[30] A. D. Dolgov and N. A. Pozdnyakov, arXiv:2009.04361.

[31] A. D. Dolgov and Ya. B. Zeldovich, Rev. Mod. Phys. 53, 1 (1981).

[32] R. Nandra, A. N. Lasenby, and M. P. Hobson, Mon. Not. R. Astron. Soc. 422, 2931 (2012).

[33] A. Chaudhuri and A. Dolgov, arXiv:2001.11219.

[34] D. N. Page, Phys. Rev. D 13, 198 (1976).

[35] S. Weinberg, The Quantum Theory of Fields (Cambridge University Press, Cambridge, England, 1995), Vol. 1 Foundation. 
[36] A. A. Starobinsky, Phys. Lett. 91B, 99 (1980); Adv. Ser. Astrophys. Cosmol. 3, 130 (1987).

[37] E. V. Arbuzova, A. D. Dolgov, and R. S. Singh, J. Cosmol. Astropart. Phys. 07 (2018) 019; 04 (2019) 014; arXiv: 2002.01931.

[38] E. V. Arbuzova, A. D. Dolgov, and R. S. Singh, Symmetry 13, 877 (2021).
[39] A. Addazi, K. Anderson, S. Ansellb et al., J. Phys. G 48, 070501 (2021).

[40] A. Ambrosone, R. Calabrese, D. F. G. Fiorillo, G. Miele, and S. Morisi, arXiv:2106.11980.

[41] D. Binosi and L. Theussl, Comput. Phys. Commun. 161, 76 (2004). 